\title{
Application of GSM for Home Devices Control
}

\author{
Amusa K. A. \\ Department of Electrical and \\ Electronic Engineering, \\ Federal University of \\ Agriculture, Abeokuta, Nigeria
}

\author{
Olanipekun M. U. \\ Department of Electrical and \\ Electronic Engineering, \\ Federal University of \\ Agriculture, Abeokuta, Nigeria
}

\author{
Nuga O. O. \\ Department of Electrical and \\ Electronic Engineering, \\ Federal University of \\ Agriculture, Abeokuta, Nigeria
}

\begin{abstract}
This paper presents an application aimed at getting more from GSM mobile phones in addition to their traditional role of voice and data communications. It involves development of a device based on application of GSM technology for the control of home appliances. The developed system has at its heart a microcontroller PIC16F877A for coordination and processing of control commands, which are in the form of short messages from mobile stations. A working prototype built for the demonstration of the design can control five connected appliances whose current consumption is not in excess of 13A. Provision is made for the display of the status of connected appliances on the LCD.
\end{abstract}

\section{General Terms}

System control

\section{Keywords}

GSM module, microcontroller, control device, home appliances.

\section{INTRODUCTION}

The new age of technology has redefined communication. Most people nowadays now have access to mobile phones and thus the world indeed has become a global village [1]. At any given moment, an individual can be contacted with the mobile phone. But the application of mobile phone cannot just be restricted to the traditional role of enabling voice conversations. Mobile data and multimedia usage are on increase as technology advances and more bandwidths are available to support the required traffics. New innovations and ideas are integrated into mobile phone as further enhancement to its capabilities. Free-space communication technologies such as Infra-Red (IR) and Bluetooth which have developed over the years have been integrated into mobile devices. All these additions and many more have contributed in no small measure to improve the quality of our life and the way we live today [1].

Remote management of several home and office appliances is a subject of growing interest and in recent years we have seen many systems being developed to provide such controls. Common remote controllers utilize IR- or Bluetoothtechnology, for communication between a controller and a controlled device. For many devices, the remote controller contains all function controls while the controlled device itself has only a handful of essential primary controls. With advances in the level of sophistication of devices and appliances, the need to extend the control range beyond those offered by IR- and Bluetooth-base controller becomes obvious.

With recent advances in telecommunications industry, new frontiers are opening for the control of appliances as most appliances now work smartly. Wireless-Fidelity (Wi-Fi) enabled switch allows control of electronic devices from distances that are longer than the reach of IR- or Bluetoothbased remote controller. Wi-Fi control is a wireless networking technology that allows computers and allied devices to communicate wirelessly within a particular area via radio waves. The Wi-Fi switch uses existing home Wi-Fi network to provide wireless control of connected devices. The only requirement is the switch has to be powered and devices to be controlled are plugged into the switch. With this arrangement, connected devices are easily controlled via use of smartphone or tablet (running Android 4.0 or higher operating systems). However, the operation of Wi-Fi control system is premised on the availability of a functional internet access and steady electricity power supply.

The Internet penetration is still very low, even among the urban dwellers not to talk of people living in villages. Devices control solutions based on application of Wi-Fi technology is thus very restricted unlike those involving Global System for Mobile (GSM) communication technology. Apart from supporting voice calls, GSM supports communication of text messages as well as multimedia messages (that may contain pictures, graphics, animations, etc.). Sending written text messages is very popular among mobile phone users as it allows quick communication and sharing of ideas, opinions and other relevant information [2]. This concept can be put into advantage via development of control system platform to send and receive messages which in fact are commands to control different appliances and devices connected to the platform.

Few accounts exist in literature where GSM technology has been successfully employed in development of controller devices. These include development of GSM-based household power management system [1], GSM-based intrusion detection and reporting system [3], SMS-based remote control system [4], DTMF-based remote control system [5], design and development of an automated home control system using mobile phone [6], GSM- based device control for remote management of home and office appliances [2], wireless load control device using GSM module [7] and SMS-based home appliance control system for automating appliances and security [8]. In all these developments either the voice prompt, short message or both capabilities of the mobile phone is utilized in the developed GSM-based control device.

GSM is an open, digital cellular technology used for transmitting mobile voice and data services. It is developed to ensure the use of the same subscriber units or mobile phone terminals throughout the world. Many GSM standards are existence such as GSM900, EGSM900, GSM1800 and GSM1900; they mainly differ only on RF carrier frequency band and bandwidth [8]. Depending on the standard, GSM supports data transfer and allows transmission of Short Message Service (SMS). This is in addition to its international 
roaming capability, allowing users to access the same services when at home or travelling abroad. One of the key features of GSM is the Subscriber Identity Module (SIM) card. The SIM is a detachable smart card containing the user's subscription information and phone book. This allows the user to retain information after swapping of mobile phones. Alternatively, the user can also change operators while retaining the handset simply by changing the SIM. It is against these capabilities of GSM that this work is embarked upon to develop a system that is based on the GSM technology that effectively enables control of devices located in a particular location from a remote area via SMS.

\section{DESIGN CONSIDERATIONS}

The design considerations for the developed GSM-based home devices control system involve two parts namely: software and hardware sections. The software part involves written and programming of the connection of the microcontroller PIC16F877A with respect to pin configurations microcontroller which occupies the heart of the developed device whilst the hardware section is concerned with activities leading to selection and choice of components that make up the totality of developed control system. Fig. 1 illustrates the developed GSM-based home appliances control system in block diagram.

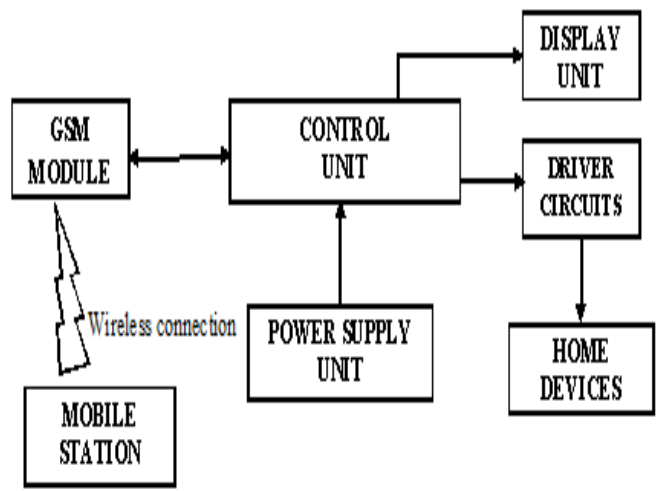

Fig. 1: Block diagram of the GSM-based home devices control

\subsection{Hardware Section}

From Fig. 1, it is obvious that the developed GSM-based home devices control system is made of the following building blocks: control unit, driver circuit, GSM module, display unit, mobile station, power supply unit and controlled home devices.

2.1.1 GSM Module: The GSM module accepts GSM network operator SIM card and acts like a mobile phone having its own unique phone number. These features pave way for its choice in this work. The GSM module employed in this work has the following specifications:
Table 1. Specifications of GSM module

\begin{tabular}{|l|l|}
\hline \multicolumn{1}{|c|}{ Features } & \multicolumn{1}{|c|}{ Specifications } \\
\hline Operating frequency & $\begin{array}{l}\text { GSM 850MHz, EGSM } \\
900 \mathrm{MHz}, \mathrm{DCS} 1800 \mathrm{MHz} \text { and } \\
\text { PCS } 1900 \mathrm{MHz}\end{array}$ \\
\hline Operating Voltage rating & $3.2 \mathrm{~V}-4.8 \mathrm{~V} \mathrm{dc}$ \\
\hline Output pin voltage & $5 \mathrm{~V} \mathrm{dc}$ \\
\hline Output pin current & $25 \mathrm{~mA}$ \\
\hline Communication mode & $\begin{array}{l}\text { UART interface, configured } \\
\text { for full-duplex asynchronous } \\
\text { mode }\end{array}$ \\
\hline Baud rate & $\begin{array}{l}\text { Supports auto bauding, } \\
9.6 \mathrm{~kb} / \mathrm{s} \text { used. }\end{array}$ \\
\hline
\end{tabular}

2.1.2 Control unit: At the heart of the developed GSM-based home-devices control system is the PIC16F877A microcontroller. The PIC16F877A microcontroller comes with a 40-pin Dual In-line Package (DIP) with internal peripherals. It is flash-based and an 8-bit microcontroller having 256 bytes of EEPROM data memory. Other features of PIC16F877A include self-programming capability, two comparators, 8 channels of 10-bit analog-to-digital converter, synchronous serial port and a UART. All of these features make it ideal for more advanced level A/D applications automotive, industrial, appliances and consumer applications.

The following pins are utilized out of the 40 that comes with the PIC16F877A: Pins 25 and 26 are configured, respectively, to interface with the transmit (TX) and receive (RX) ports of the GSM modem; pins 20, 21, 22, 27 and 28 interface with driver circuit to effectively control the intended devices. Pin 19 is programmed to effectively switch ON or OFF the GSM modem.

Crystal oscillator is used to generate clock and provide frequency stability to the microcontroller. Its terminals are connected to the PIN 13 and PIN 14 of the microcontroller to drive the device. Two capacitors $\mathrm{C} 1$ and $\mathrm{C} 2$, each with a value of $22 \mathrm{pF}$, act as the load capacitance of the crystal and for smoothening of the clock pulses. The frequency of the crystal oscillator used is $20 \mathrm{MHz}$. Fig. 2 depicts the circuit.

2.1.3 Driver circuit: output pins of the PIC16F877A are all at $5 \mathrm{Vdc}$, which is not enough to power most of the intended home devices and appliances. Consequently, there is need for driver circuits to interface the PIC16F877A on one hand and the target home appliance on the other.

In this work, the driver circuit employed is shown in Fig. 3 where a PNP transistor (C1815) is used as a buffer to drive the relay. The relay is QUAZ-SH-105L. The output pin of the PIC16F877A is interfaced with the $\mathrm{C} 1815$ via $10 k \Omega$. Since the microcontroller can provide only $25 \mathrm{~mA}$ at its output, which is not sufficient enough to energize the relay coil that requires approximately $100 \mathrm{~mA}$, transistor $\mathrm{C} 1815$ is utilized to buffer the current. 


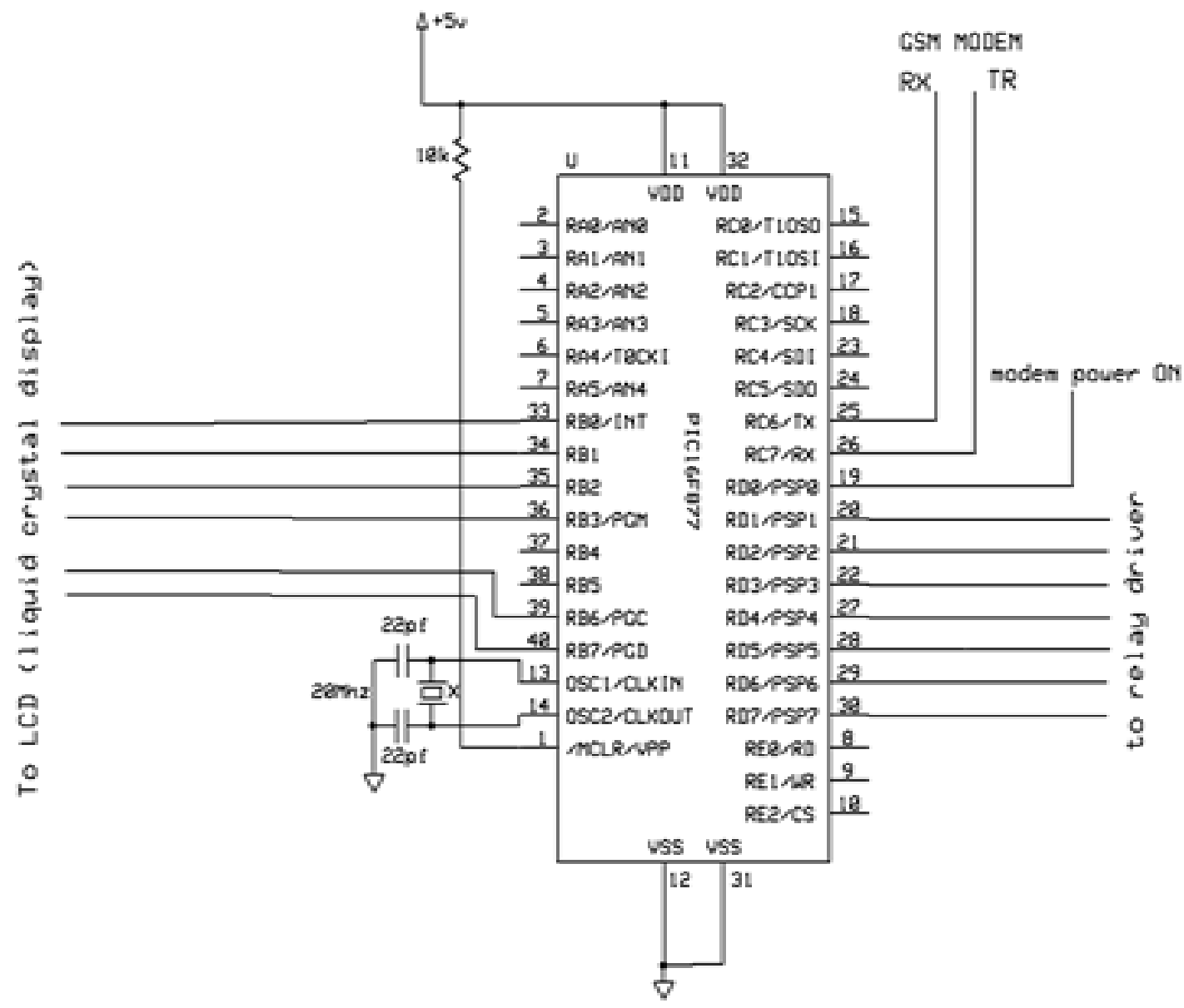

Fig. 2: Pin configuration of PIC16F877A as utilized in the developed GSM-based home devices control

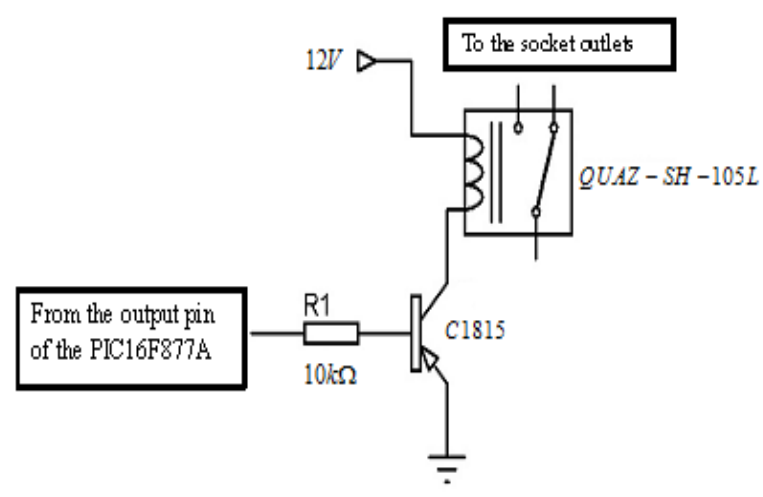

Fig. 3: Circuit diagram of the driver circuit

2.1.4 Display unit: The display unit is made of $16 \times 2$ Liquid Crystal Display (LCD) module for the display of short messages in the form of textual information to the user on the operation status of the developed GSM-based home appliances controller. The LCD is interfaced with PIC16F887A through port B of the microcontroller. Information to be displayed is sent through pins 39 and 40, corresponding to PGD and PGC pins of the microcontroller, respectively. It is put into operation by a regulated $5 \mathrm{~V}$ as well as its backlight. The backlight contrast is controlled through pin 3 of the LCD via a variable resistor of $10 \mathrm{k} \Omega$. The following pins are utilized out of the 16 that come with LCD: pin 1 (Ground), pin $2(+5 \mathrm{~V})$, pin 3 (VLC), pin 4 (RS), pin 5 (RD), pin 6 (EN), pins $7-10$ (un-used), pin 11 (D4), pin 12 (D5), pin 13 (D6), pin 14 (D7), pin 15 (anode of backlight) and pin 16 (cathode of backlight). The connection of the LCD to the microcontroller is shown in the Fig. 4.

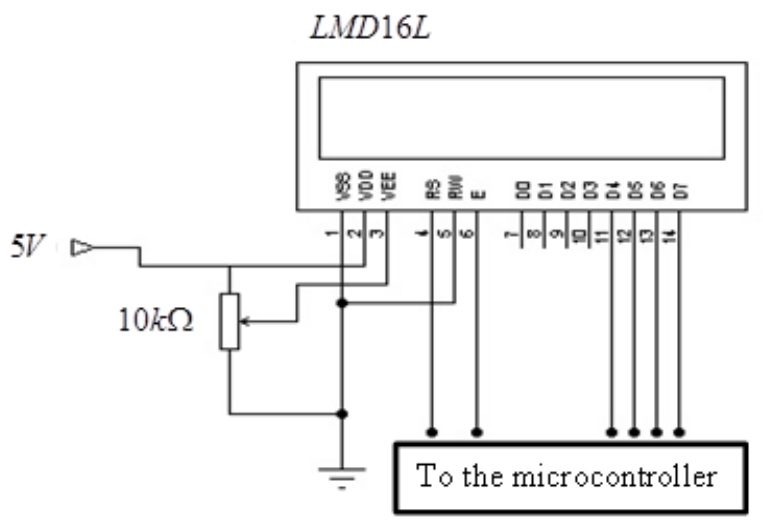

Fig. 4: Circuit diagram of the LCD module 
2.1.5 Power supply unit: The developed device is powered from the regulated power supply that is extracted from the main supply. The unit has a $220 \mathrm{~V} / 12 \mathrm{~V}$ step down transformer, the output of which is rectified using bridged rectifier and regulated to $5 \mathrm{~V}$ via $\mathrm{LM} 7805$ needed by PIC16F877A.

2.1.6 Mobile station: The represents mobile phone or mobile terminal with a functional GSM SIM card through which communication is established with the developed GSM-based home devices control system. Control signals are generated through the mobile station in order to ensure the needed control on connected devices.

2.1.7 Home devices: These are controlled devices. These devices are connected to output of the developed GSM-based control system and are permanently switched ON from the accompanied switch. In this work, provision is made for the control of five different devices, each of which current consumption is not more than 13A.

\subsection{Software Phase}

The software phase involves programming of the PIC16F877A microcontroller. The microcontroller can be programmed using $\mathrm{C}, \mathrm{C}++$ and assembly language. For the developed GSM-based home devices control system, the firmware PIC16F877A is developed in assembly language and compiled with MPLAB assembler.

\section{IMPLEMENTATION}

Fig. 5 illustrates the complete circuit diagram of the developed GSM-based home devices control system. The developed GSM-based home devices control system is implemented by assembling components in line with the circuit diagram presented in Fig. 5. The microcontroller PIC16F877A has the written assembly code assembled on it via use of MPLAB. The entire construction is powered by the power supply unit. The programming event is such that the microcontroller reads the command in the form of short messages from the mobile station

If the command specifies ON for a particular home device, the microcontroller gives a high output for the relay attached to the device and sends message about the status to the mobile station that sends message to the mobile station.

Table 2. Control messages and interpretations

\begin{tabular}{|c|l|}
\hline Message sent & \multicolumn{1}{|c|}{ Interpretation/Action } \\
\hline "1 on" & Device 1 is switched on \\
\hline "1 off" & Device 1 is switched off \\
\hline "2 on" & Device 2 is switched on \\
\hline "2 off" & Device 2 is switched off \\
\hline "3 on" & Device 3 is switched on \\
\hline "3 off" & Device 3 is switched off \\
\hline "4 on" & Device 4 is switched on \\
\hline "4 off" & Device 4 is switched off \\
\hline "5 on" & Device 5 is switched on \\
\hline "5 off" & Device 5 is switched off \\
\hline "all" & All devices are switched on \\
\hline "clear" & All devices are switched off \\
\hline "status" & Check status of connected devices \\
\hline
\end{tabular}

A $220 \mathrm{~V}$ ac volt is connected to the Normally Open (NO) terminal of the relay while the Normally Closed (NC) one is connected to the positive terminal of the device to be powered. This is to ensure that when the output pin of PIC16F877A gives a high output to the input of the relay; the electromagnet of the relay is energized thereby attracting the connecting pole to turn $\mathrm{NO}$ to $\mathrm{NC}$ and thereby power $\mathrm{ON}$ the connected device. The connection remains ON until the PIC16F877A receives command to OFF the affected device.

Table 2 presents format of messages (commands) for the control of the connected devices to the developed GSM-based home device control

The commands (messages) for the developed GSM-based home devices control system are not case sensitive. Therefore, text messages are either composed using upper case or lower case English alphabets.

\section{TEST AND RESULTS}

After the construction and implementation of the designed circuit, a number of tests are conducted to ascertain that it works to specifications. Loads to be controlled and monitored are connected to outputs of the developed GSM-based home appliances control. In all, five different appliances whose current rating is not more than $13 \mathrm{~A}$ are utilized in the test. A laptop computer system is connected to the socket labeled 1 , an electric kettle to socket 2, socket 3 powers an electric lamp, a phone charger is supplied by socket 4 while an electric blender is attached to socket 5. Fig. 6 presents a snap shot of the set-up for the tests. The developed GSM-based home devices control system is connected to the mains. The following tests are carried out on the constructed device:

i) Initialization test on developed device

ii) Status check of the device

iii) Switching OFF all loads from ON condition

iv) Switching ON all loads from OFF condition

v) Switching OFF an appliance

vi) Switching $\mathrm{ON}$ an appliance

vii) Response time for sent messages

\subsection{Initialization of the device}

When the developed GSM-based home device control system is powered $\mathrm{ON}$ via connection to the mains, the LCD displays GSM BASED DEVICE CONTROL. The displayed message changes to DEVICE READY after elapse of five seconds and then shows the status of the device as OUTPUT LOADS 12345 after further five seconds timeout. Each of the developed GSM-based home devices control system output ports are checked with the Voltmeter. The voltage reading in each case is $220 \mathrm{~V}$, which is enough to power home appliances. Fig. 7 shows snap shots of the displays during initialization of the developed GSM-based home devices control system.

\subsection{Status check of the device}

Each of the connected appliances switch is put ON, the mains switch to the developed GSM-based device control system is put ON. The developed device is then powered ON via the control switch on it. The LCD displays GSM BASED DEVICE CONTROL then it changes to DEVICE READY in five seconds. All connected appliances become operational as they are powered. Ignoring the status message shown on the display unit, which is OUTPUT LOADS 12345, an SMS message STATUS is sent from the mobile station to communicate with the developed GSM-based home devices control system. On reception of the SMS message, the LCD displayed MESSAGE RECEIVED and then OUTPUT LOADS 12345. Almost immediately, the device replies with an SMS message OUTPUT LOADS 12345 to the mobile station from which the initial message is generated, indicating that all output ports of the developed control system are ON. Fig. 8 illustrates snap shots of the last three stages of the procedure involved. 


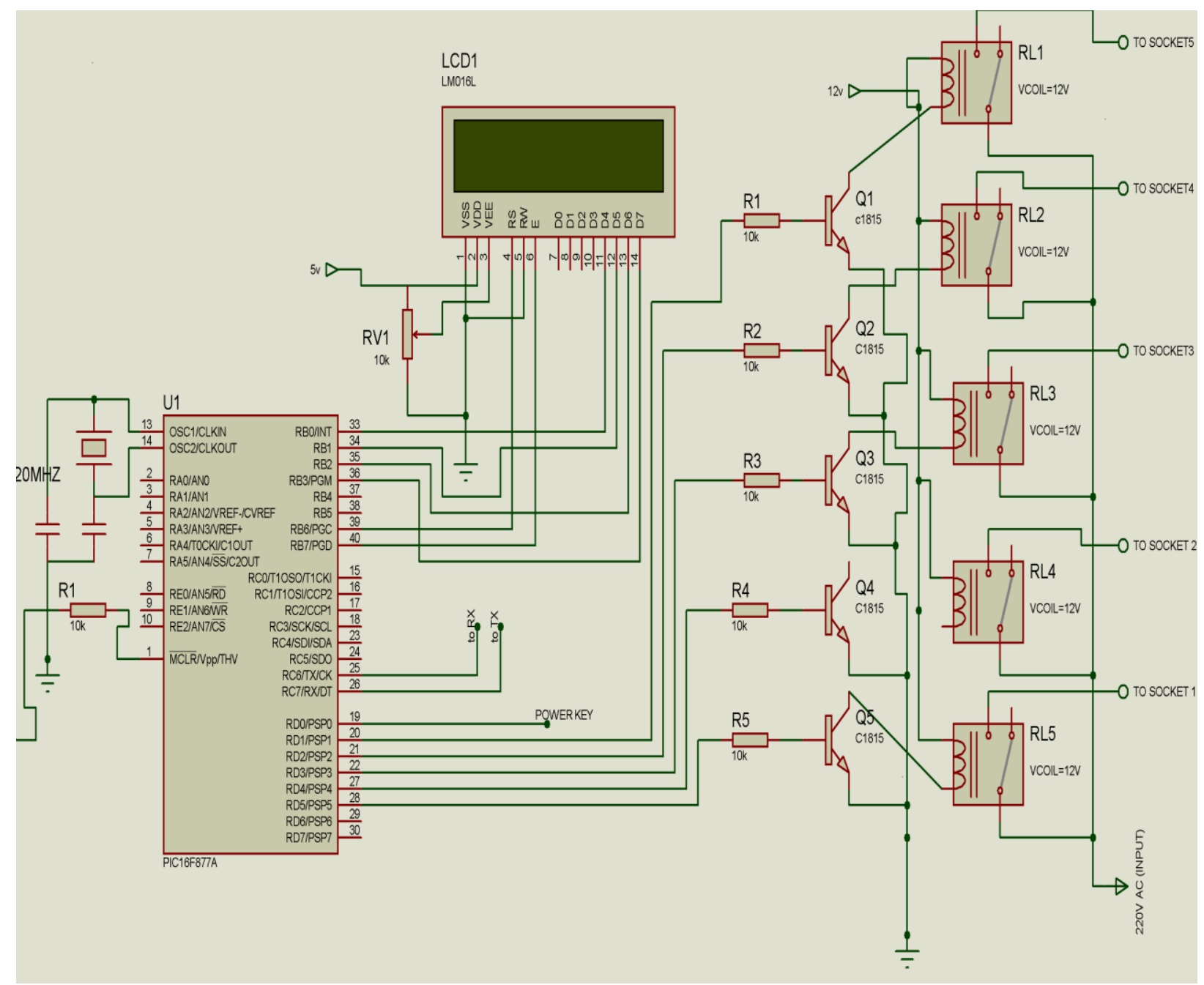

Fig. 5: Circuit diagram of the GSM-based home devices control system

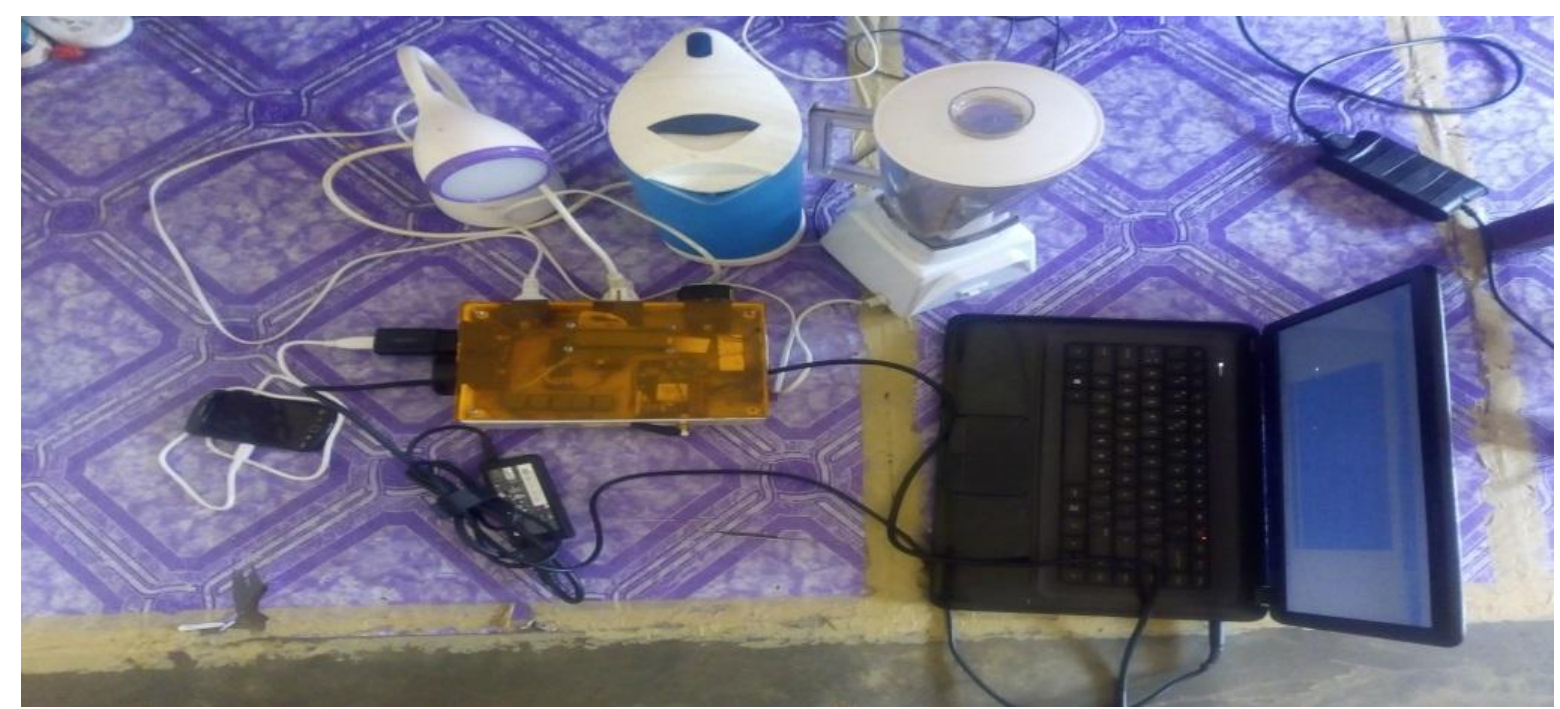

Fig. 6: Set-up for connected loads during testing of the developed GSM-based home devices control system 


\subsection{Switching OFF of all loads from ON conditions}

An SMS message CLEAR sent from the mobile station to the developed GSM-based home devices control system activates the device to switch OFF all its output ports. The LCD on the device displays first MESSAGE RECEIVED and then responded by switching off all loads after which the displayed message turns to OUTPUT LOADS, 00000. The five zeros indicates that all loads are not powered and in off position. Snap shots of the process is presented in Fig. 9.

\subsection{Switching ON of all loads from OFF condition}

Each of the connected appliances switch is put ON, followed by that of the mains switch to the developed device control system. After the initialization process, the LCD displays OUTPUT LOADS, 00000, indicating that all output sockets are inactive. A command message ALL is sent from the mobile station to the developed GSM-based control device to switch ON all its output ports. The system acknowledges the receipt of the sent message via display of MESSAGE RECEIVED and then OUTPUT LOADS 12345. Simultaneously, the developed control device sends a response message OUTPUT LOADS, 12345 back to the mobile station from the command message is originated. Illustration of the process is captured in Fig. 10

\subsection{Switching OFF an appliance}

Assuming the developed GSM-based control system is fully initialized with all of the connected appliances switched ON. The LCD under this condition displays OUTPUT LOADS, 12345, indicating that all sockets are active. Then to switch OFF a desired appliance say the appliance connected to output port 2 with other output ports (sockets 1, 3, 4 and 5) remain active, a SMS message $\mathbf{2 O F F}$ is sent from the mobile station to the developed GSM-based home device control system to put OFF the output port 2. The developed system responded and rendered its port 2 inactive leaving ON output ports 1, 3, 4 and 5. Snap shots peculiar to this test is shown in Fig. 11.

\subsection{Switching ON an appliance}

Suppose the test carried out in the preceding section where port 2 of the GSM-based device control is inactive is to be reversed such that port 2 is to be active along with others. A status check reveals that sockets $1,3,4$ and 5 are active while port 2 is not. To switch $\mathrm{ON}$ a desired appliance say the appliance connected to output port 2 while all other output ports $1,3,4$ and 5 remain ON. A SMS message $\mathbf{2 O N}$ is sent to the developed from the mobile station to switch ON output port 2. This test is demonstrated in Fig. 12.
4.7 Message delivery and delivery time test This test is carried out to determine time taken by the developed GSM-based home devices control system to respond to command issued from the mobile station. Although, the message delivery time is a function of the quality of service offered by the telecoms operators' whose SIM cards are utilized for communication. However, it is instructive to ascertain which of the operator offers better services in the area where the developed GSM-based home devices control system is to be deployed. The test is conducted at The test is conducted at the Applied Electricity Lab, Electrical Electronics Engineering Department, Federal University of Agriculture, Abeokuta. The time taken by different communication networks to deliver the sent message is recorded. The SIM card in the GSM modem is changed in turn while carrying out this test. The duration of each test is a function of the time taken for message delivery. For a particular operator SIM card, the test is repeated at three different times: 8am, 12noon and 4pm. The choice of the time for the test is informed by the relative distribution of traffic on GSM networks with respect to peak- and off-peak-hour periods. The readings obtained for three different networks out of the four available in Federal University of Agriculture, Abeokuta are presented in Table 3.

Table 3: Delivery time of short messages sent by GSM module

\begin{tabular}{|c|c|c|c|}
\hline $\begin{array}{l}\text { Network } \\
\text { Provider }\end{array}$ & $\begin{array}{l}\text { Time of } \\
\text { the Test }\end{array}$ & $\begin{array}{l}\text { Message } \\
\text { delivery } \\
\text { Time (s) }\end{array}$ & $\begin{array}{c}\text { Average } \\
\text { delivery Time } \\
\text { (s) }\end{array}$ \\
\hline \multirow{3}{*}{ A } & 6am & 33 & \multirow{3}{*}{31} \\
\hline & $12 \mathrm{pm}$ & 21 & \\
\hline & $4 \mathrm{pm}$ & 38 & \\
\hline \multirow{3}{*}{ B } & 6am & 29 & \multirow{3}{*}{30} \\
\hline & $12 \mathrm{pm}$ & 21 & \\
\hline & $4 \mathrm{pm}$ & 40 & \\
\hline \multirow{3}{*}{$\mathrm{C}$} & $6 a m$ & 32 & \multirow{3}{*}{33} \\
\hline & $12 \mathrm{pm}$ & 22 & \\
\hline & $4 \mathrm{pm}$ & 45 & \\
\hline
\end{tabular}

It can be inferred from Table 3 that, as at the time of conducting the message delivery time test on the developed GSM-based home appliances control system at Federal University of Agriculture, Abeokuta, the communication network B performs better than the other two. The implication of this result is that periodically, the user should check and ascertain the average message delivery time in order to decide the preferred SIM card to be inserted in the GSM module. 


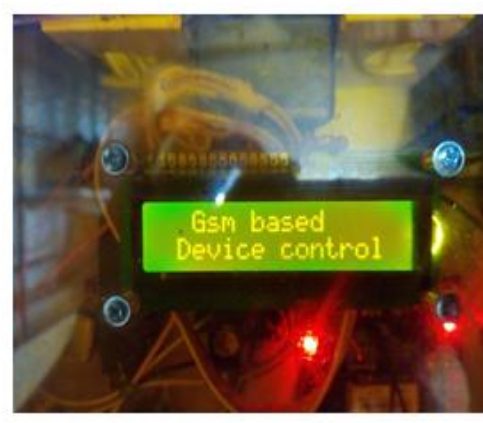

(a)

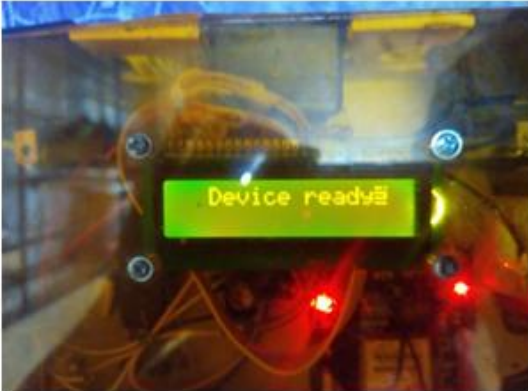

(b)

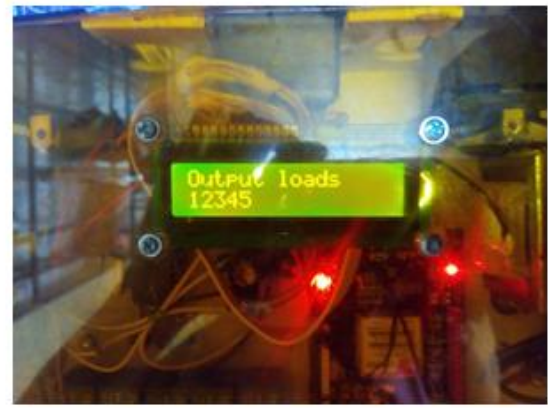

(c)

Fig. 7: Snap shots showing the initialization process of the developed GSM-based home devices control system (a) power ON message (b) message displayed 5 seconds after power ON (c) message displayed 10 seconds after power ON

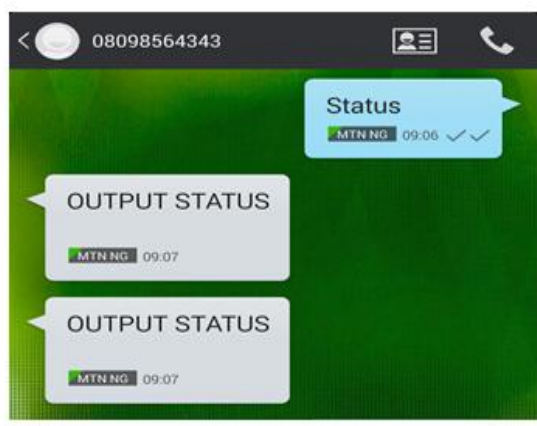

(a)

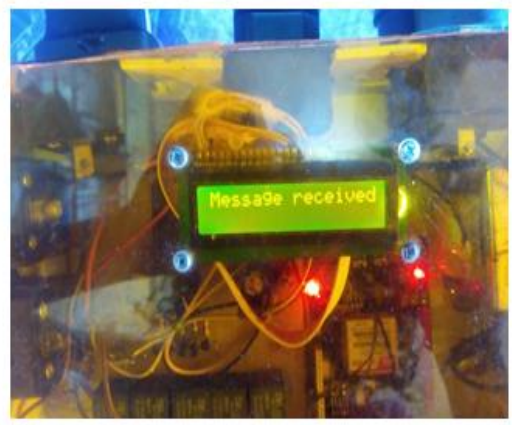

(b)

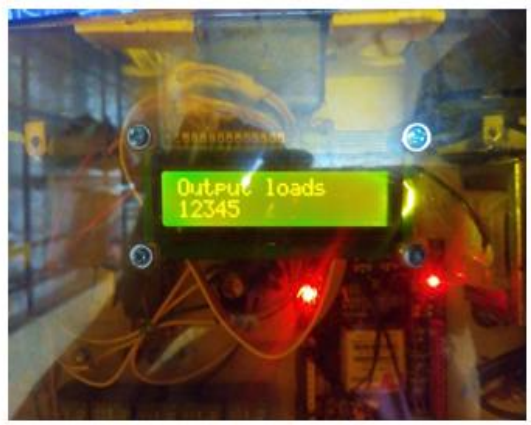

(c)

Fig. 8: Snap shots showing status check on output ports of the developed GSM-based home devices control system (a) message sent from the mobile station (b) acknowledgement of the received message (c) response message to the mobile station

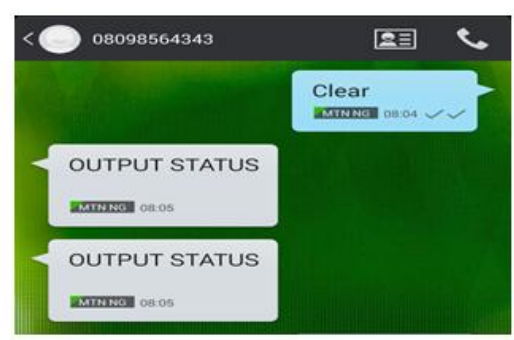

(a)

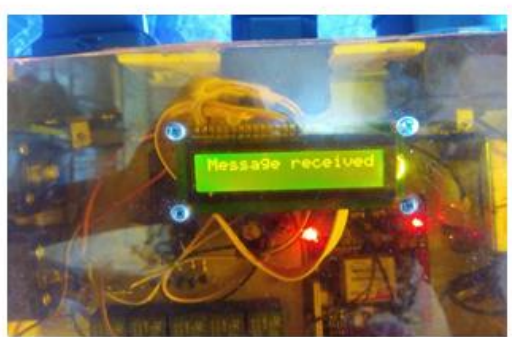

(b)

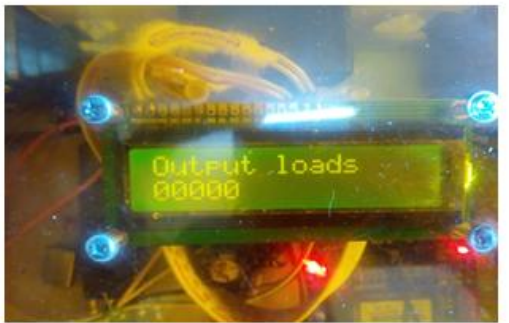

(c)

Fig. 9: Snap shots showing switching off of all output ports of the developed GSM-based home devices control system (a) message sent from the mobile station (b) acknowledgement of the received message (c) response message to the mobile station

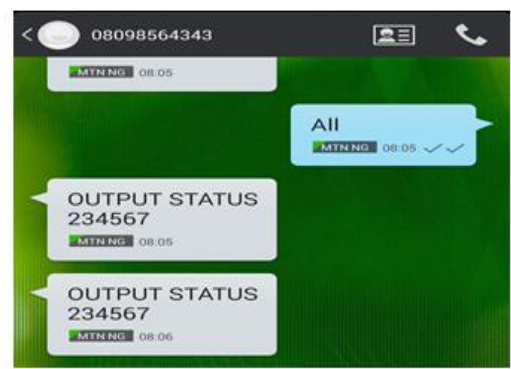

(a)

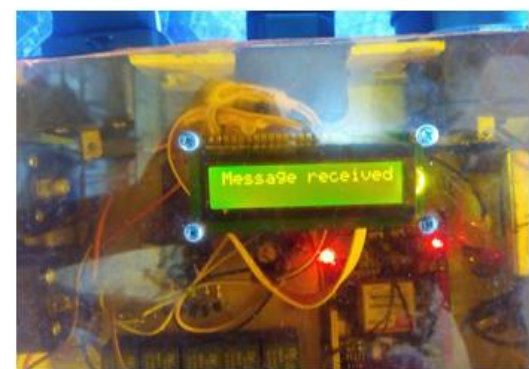

(b)

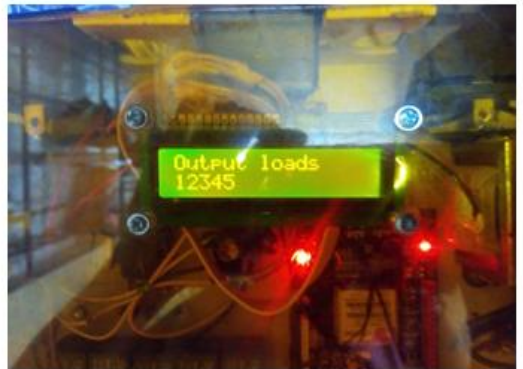

(c)

Fig. 10: Snap shots showing switching on of all output ports of the developed GSM-based home devices control system (a) message sent from the mobile station (b) acknowledgement of the received message (c) response message to the mobile station 


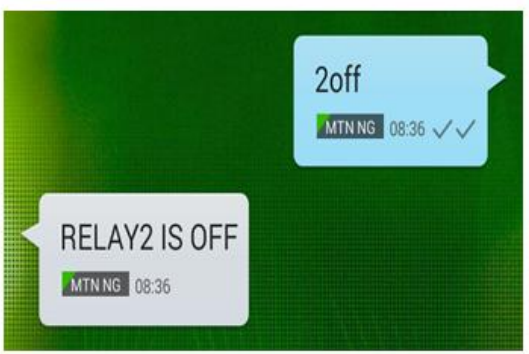

(a)

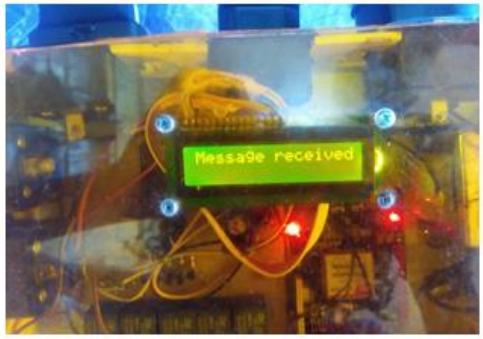

(b)

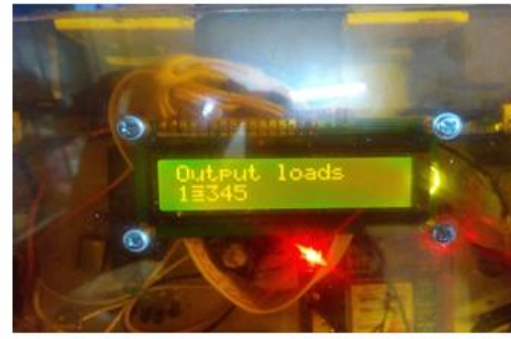

(c)

Fig. 11: Snap shots showing switching off of the output port 2 of the developed GSM-based home devices control system (a) message sent and message received by the mobile station (b) acknowledgement of the received message (c) response message sent to the mobile station

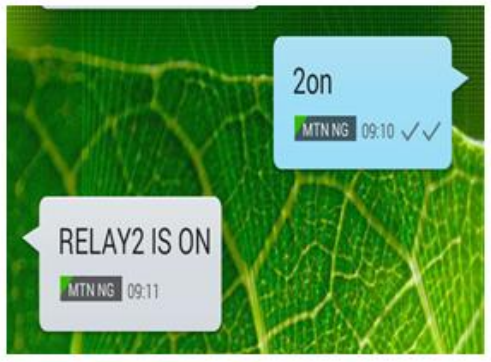

(a)

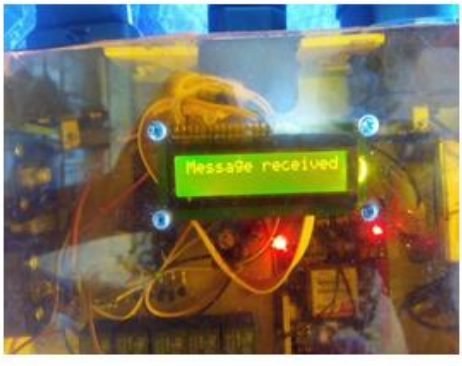

(b)

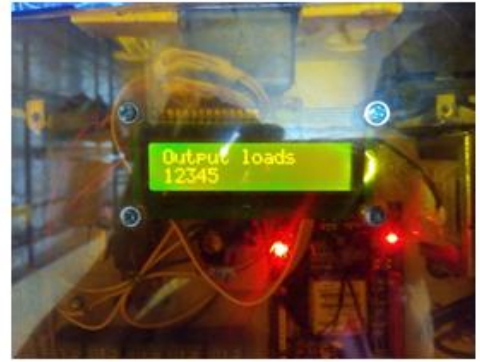

(c)

Fig. 12: Snap shots showing switching on of the output port 2 of the developed GSM-based home devices control system (a) message sent and message received by the mobile station (b) acknowledgement of the received message (c) response message sent to the mobile station

\section{CONCLUSION}

This paper focuses on development of GSM-based home appliances control system appliances with wireless technology which has revolutionized our way of living. Technique used in this system is not complex and generally available. The extensive capabilities of this system are what make it so interesting. From the convenience of a simple cell phone, a user is able to control and monitor virtually any home electrical devices connected to the developed system. The need to physically present in any location for tasks involving the operation of electrical appliances within a household is eliminated. In addition, the developed system is an open platform in the sense that any GSM SIM card belonging to any of the available GSM service providers can be utilized to establish communication between the mobile station and the developed home appliances control system. The concept of employed in this work could be deployed and adapted in areas such as, remote sensing, robotics, aeronautics, home automation, and many others where continuous monitoring and regulation is needed. Furthermore, the present development can be improved upon such that home appliances are effectively controlled via voice command or combination of both voice and short messages.

\section{REFERENCES}

[1] Ganiyu R.A., Arulogun O.T., Adetunji A.B. and O. O. Okediran. 2011. Development of GSM Based household power management System. British Journal of Science 1(2):1-9.
[2] Awodele O., B. A. Olugore, A. A. Izang and A. Adams. 2011. Multiple Unit GSM Controlled Devices. International Journal of Computer Trends and Technology (IJCTT). 1(1):61-69.

[3] K.A. Amusa, A. Adewusi, O.O. Nuga, A.J. Olanipekun \& O.A. Adewale (2015): Pyro-Electric Infrared SensorBased Intrusion Detection and Reporting System. Afr J. of Comp \& ICTs. 8(2): 91-98.

[4] Amit C. and Reecha R.S. 2011. SMS Based Remote Control System. International Journal of Computer Science, 2(2):39 - 42.

[5] Sharma R., K. Kumar, and S. Viq. 2006. DTMF Based Remote Control System, IEEE International Conference ICIT 2006, 2380-2383, December

[6] Shoewu, L.A, Makanjuola, N.T and A. A. Ajasa. 2014. Design and Development of an Automated Home Control System Using Mobile Phone. World Journal of Control Science and Engineering, 2 (1):16-11.

[7] Quitrakul S. and S. Watanasophon. 2014. Wireless Load Control Device using GSM Module. $3^{\text {rd }}$ Int. Conf. on Informatics, Environment, Energy and Applications. 66:87-91.

[8] Malik, S. H. K., Aihab, K. and S. Erum. 2009. SMS Based Home Appliance Control System for Automating Appliances and Security. Issues in Informing Science and Information Technology 6: 887-894. 